\title{
Homogeneity improvement of field emission beam from metallic nano-tip arrays by noble-gas conditioning
}

\author{
S. Tsujino, ${ }^{1, a)}$ M. Paraliev, ${ }^{2}$ E. Kirk, ${ }^{1}$ and H.-H. Braun ${ }^{3}$ \\ ${ }^{1}$ Laboratory for Micro- and Nanotechnology, Department of Synchrotron Radiation and Nanotechnology, \\ Paul Scherrer Institut, Villigen-PSI CH-5232, Switzerland \\ ${ }^{2}$ Department of Large Research Facilities, Paul Scherrer Institut, Villigen-PSI CH-5232, Switzerland \\ ${ }^{3}$ SwissFEL, Paul Scherrer Institut, Villigen-PSI CH-5232, Switzerland
}

(Received 14 April 2011; accepted 20 July 2011; published online 15 August 2011)

\begin{abstract}
The authors explored control of field-emission electron beam homogeneity emitted from metallic nano-tips fabricated by molding. Improved beam homogeneity and increased maximum bunch charge were observed after DC operation of cathodes in low-pressure argon gas environment. They also observed average current per tip of $4.5 \mu \mathrm{A}$ from a $1 \times 10^{4}$ emitter array device after conditioning in neon gas. Evolution of Fowler-Nordheim parameters as a result of noble-gas conditioning was discussed. (C) 2011 American Institute of Physics. [doi:10.1063/1.3624705]
\end{abstract}

Field-emitter array (FEA) cathodes with high emission current are promising for applications that require high beam brightness such as free electron lasers ${ }^{1,2}$ and microwave/THz vacuum electronic amplifiers. Using metallic FEAs fabricated by molding, ${ }^{3}$ sub-nanosecond electrical switching in high acceleration electric field up to $30 \mathrm{MV} / \mathrm{m}$ [Refs. 1 and 2] and ultrafast near infrared laser-induced field emission with up to $5 \mathrm{pC}$ electron charge pulse [Ref. 4] were demonstrated recently. However, field emission beam imaging experiments $^{1,2}$ indicated that only a limited number of tips emitted electrons. This is partly because of the distribution of the tip radii of curvature $R_{\text {tip }}{ }^{5-8}$ that demands a procedure to improve it. This is particularly important for high current operation, since, when gate voltage is increased for higher current, current density at sharp emitters that are already switched on at low-voltages, could easily exceed the critical value in the order of $10^{7}-10^{8} \mathrm{~A} \mathrm{~cm}^{-2}$ and trigger destructive vacuum arc. ${ }^{9}$ In this letter, aiming at homogenizing the $R_{\text {tip }}$ distribution, we explored the effect of in-situ exposure of FEA tips to low pressure noble gas and studied evolution of beam image and current-voltage $(I-V)$ characteristics.

Three single-gate FEAs, FEA1 $\left(5 \mu \mathrm{m}\right.$-pitch, $1.2 \times 10^{5}$ tips), FEA2 $\left(5 \mu \mathrm{m}\right.$-pitch, $3 \times 10^{4}$-tips $)$, and FEA3 $(10 \mu \mathrm{m}$ pitch, $1 \times 10^{4}$-tips) were fabricated by molding and a self-aligned gate process. $^{3}$ These consisted of pyramidal shape molybdenum field emitters with nominal $R_{\text {tip }}$ of $\sim 5$ $\mathrm{nm}, \sim 1.2-\mu \mathrm{m}$-height, and $1.5-\mu \mathrm{m}$-base-size. Inscribed circle diameters $D$ of the gate aperture holes were $\sim 2 \mu \mathrm{m}$. To observe the field emission beam image, we integrated FEAs in a high-voltage diode gun of SwissFEL gun test facility. ${ }^{1,2}$ In the diode gun, a negative $200 \mathrm{keV}$ pulse with 250 ns duration was applied to the cathode. Field emission electron pulses from FEAs with $\sim 0.5$ ns duration were synchronously generated when the cathode voltage was at the largest. The field emission switching was done by applying negative potential $-V_{\text {ge }}$ to the emitter substrate with respect to the gate electrode, which was in contact to the cathode holder of the gun. ${ }^{1,2} V_{\text {ge }}$ consisted of a DC bias and a $\sim 1$ ns pulse. DC bias was kept small for the imaging experiment (with DC

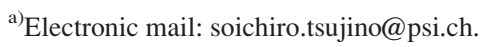

emission current below $\left.\sim 10^{-5} \mathrm{~A}\right)$. The field emission beam image was detected by a scintillating screen. Pulse charge was measured by inserting a Faraday-cup. Before the diode gun experiment, we first conditioned FEAs in a preparation chamber. The initial conditioning ${ }^{2,10}$ involved a repeated DC-bias scan or an application of a $50 \mathrm{~Hz}-\mathrm{AC}-V_{\mathrm{ge}}$ at the base pressure of $(1-5) \times 10^{-9}$ mbar while slowly increasing the maximum $V_{\mathrm{ge}}$ over a few days until $I-V \mathrm{~s}$ became stable. During the conditioning in the preparation chamber, we collected electrons by an anode biased at a positive voltage $V_{\mathrm{a}}$ of $1 \mathrm{kV}$, while measuring the anode current $I_{\mathrm{a}}$, the gate current $I_{\mathrm{g}}$, and the current $I_{\mathrm{em}}$ injected into the emitter substrates. We then transferred FEAs to the diode gun through air, and again DC conditioned those at the base pressure of $(0.5-1) \times 10^{-8}$ mbar. In the diode gun, we were able to monitor only $I_{\mathrm{em}}$ during conditioning with DC $V_{\mathrm{ge}}$ with no additional potential between cathode and anode. ${ }^{11}$

In Figure 1(a), we show an image of a 0.5 ns-long field emission pulse from FEA1 with $0.7 \mathrm{pC}$ charge and $200 \mathrm{keV}$ beam energy. $V_{\text {ge }}$ of $78 \mathrm{~V}$ (DC $45 \mathrm{~V}$ superposed by $33 \mathrm{~V}$ pulse) was applied to generate the pulses. Granular spots indicated that beam was emitted from a small number of emitters. $^{2}$ Attempted pulsed-current tip-blunting ${ }^{8,12}$ by application of higher $V_{\text {ge }}$ pulses was not successful, leading to a partial loss of emitters (approximately one half) by vacuum arc. We, therefore, tested Ar gas effect on FEAs. For that, we stopped the acceleration voltage and $V_{\text {ge }}$ pulse and applied a DC $V_{\text {ge }}$ up to $80 \mathrm{~V}$ to the chip under Ar gas pressure of $10^{-4}$ mbar for $2 \mathrm{~h}$. This resulted in a gradual increase of $I_{\mathrm{em}}$ to $\sim 7 \mathrm{~mA}$ at 80 $\mathrm{V}$ as shown in Fig. 1(d). The increased conductance near zero $V_{\text {ge }}$ showed that $I_{\mathrm{g}}$ increased substantially. Nevertheless, we were able to extract $0.7 \mathrm{pC}$ of bunch charge at $V_{\mathrm{ge}}$ of $83 \mathrm{~V}$ (DC $50 \mathrm{~V}$ and $33 \mathrm{~V}$ in pulse) as shown in Fig. 1(b). This was acquired with the same gun and camera setting as in Fig. 1(a) and displayed with the same contrast scale. Although granular emission pattern persisted, emission emerged from some areas (near bottom edges) where it was absent before the Ar conditioning. Also less bright spots were observed. To quantify this beam homogenization effect, we compared normalized and angle-averaged autocorrelation $f_{\mathrm{ac}}(r)(r$ is the spatial scale on the screen) as shown in Fig. 1(c). We found that the width 

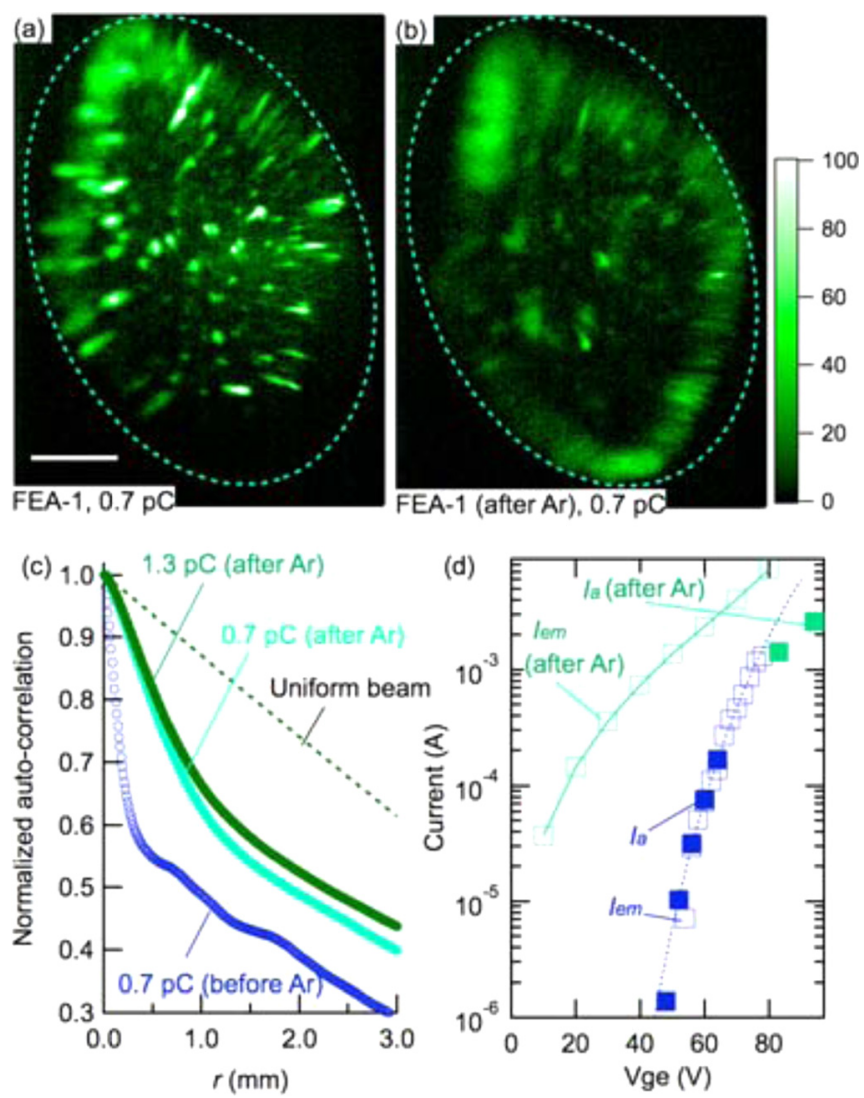

FIG. 1. (Color online) Beam images of 0.5 ns-long field-emission electron pulses from FEA1 with $0.7 \mathrm{pC}$ bunch charge, (a) before and (b) after Ar conditioning. The bar in (a) corresponds to $2 \mathrm{~mm}$ on the scintillating screen. (c) Autocorrelation of beam images shown in (a) and (b), together with that of a $1.3 \mathrm{pC}$ beam. The curve shows a simulated autocorrelation for a uniform beam with the elliptic beam envelope (broken curves in (a) and (b)). (d) $I-V \mathrm{~s}$ of FEA1, before and after Ar conditioning. Open marks show $I_{\mathrm{em}}$ (current injected to the emitter substrate) measured with DC $V_{\text {ge }}$. Filled marks indicated actual emission current $I_{\mathrm{a}}$ as evaluated from the bunch charge of the accelerated beam pulses divided by the pulse duration of $0.5 \mathrm{~ns}$.

$\left.r\right|_{70 \%}$ increased 4 times after the Ar conditioning (we defined $\left.r\right|_{70 \%}$ by $\left.f_{\text {ac }}\left(\left.r\right|_{70 \%}\right)=70 \%\right)$. It was $32 \%$ of the $f_{\text {ac }}$-width of an elliptic uniform beam with the envelope indicated by broken curves in Fig. 1. In addition, we were able to increase $V_{\text {ge }}$ to $94 \mathrm{~V}$ (DC $61 \mathrm{~V}$ and $33 \mathrm{~V}$ in pulse) to produce electron pulses with $1.3 \mathrm{pC}$ bunch charge, which exhibited the same $\left.r\right|_{70 \%}$ width as the $0.7 \mathrm{pC}$ bunch charge beam [Fig. 1(c)]. In contrast, we observed no effect when nanosecond pulses drove a FEA for $3 \mathrm{~h}$ with $10 \mathrm{~Hz}$ repetition rate under the same $\mathrm{Ar}$ gas pressure.

Aiming at realizing more homogeneous field emission beam, we next repeated experiment with FEA2 but with careful conditioning so as not to lose any emitter tips by vacuum arc before beam imaging. In particular, we kept DC $V_{\mathrm{ge}}$ below $\sim 50 \mathrm{~V}$ during conditioning in the diode gun, which avoided the increase of $I_{\mathrm{g}}$. In Fig. 2(a), we show the beam image of FEA2 with 2 pC charge and $200 \mathrm{keV}$ beam energy. The beam homogeneity and the beam current density were higher than FEA1. The autocorrelation $\left.r\right|_{70 \%}$-width of the beam was $57 \%$ of a uniform elliptic beam (broken curve of Fig. 2(a)).

To understand the Ar gas conditioning process on FEA2 quantitatively, we examined evolution of $I-V \mathrm{~s}, a$ to $d$ in Fig.
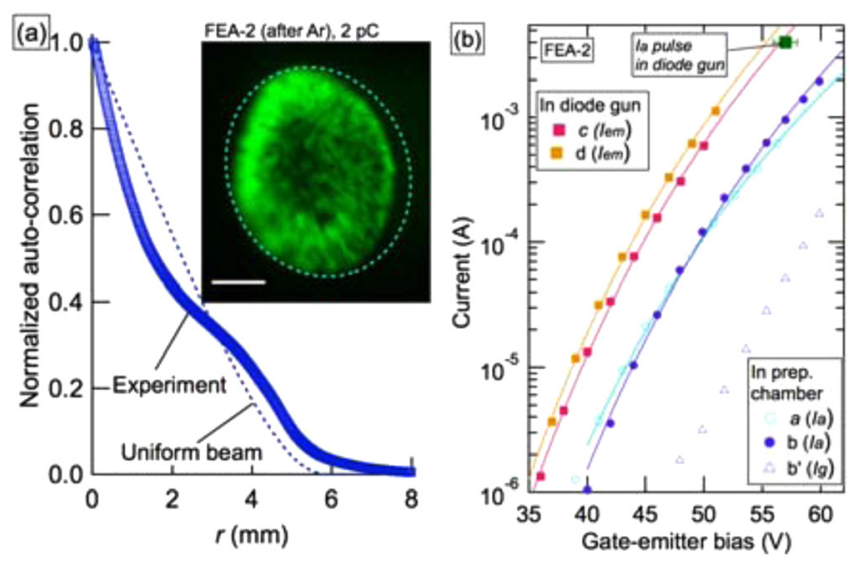

FIG. 2. (Color online) (a) Field emission image of FEA2 with 2 pC charge and $200 \mathrm{keV}$ beam energy together with its autocorrelation. The curve shows a simulated autocorrelation of a uniform beam with elliptic beam envelope (broken curve in the inset) (b) DC and pulsed $I-V \mathrm{~s}$ of FEA2 in the preparation chamber and in the diode gun.

2(b), before the imaging experiment (in the gun, $I_{\mathrm{a}}$ was approximated by $\left.I_{e m}\right)$. The data were fitted well by a function (curves in Fig. 2(b)), ${ }^{13,14}$

$$
I_{a}=A_{F N}\left(V_{g e} / B_{F N}\right)^{n} \exp \left(-B_{F N} / V_{g e}\right)
$$

with $n$ equal to 2 . The fitting parameters $A_{F N}$ and $B_{F N}$ were summarized in Table I. This function is obtained from the Fowler-Nordheim (FN) approximation of $I_{\mathrm{a}}$

$$
I_{a}=S\left(a e^{b c^{2} / W^{1 / 2}} / W\right) F^{2} \exp \left(-b W^{3 / 2} / F\right)
$$

where $S$ is the total emission area given by $\sim N R_{\text {tip }}{ }^{2}$ ( $N$ is the average number of current-emitting tips), $F$ is the electric field at the emitter apex, $W$ is the work function, and $a b$, and $c$ are parameters equal to $1.541434 \times 10^{-6} \mathrm{~A} \mathrm{eV} \mathrm{V}^{-2} 6.830890$ $\mathrm{eV}^{-3 / 2} \mathrm{~V} \mathrm{~nm}^{-1}$ and $1.199985 \mathrm{eV} \mathrm{V}^{-1 / 2} \mathrm{~nm}^{-1 / 2}$ respectively. ${ }^{13,14}$ From Eqs. (1) and (2), one finds that $A_{F N}$ is proportional to $N R_{\text {tip }}{ }^{2} W^{2} e^{b c^{2} / W^{I / 2}} . B_{F N}$ is proportional to $W^{3 / 2}\left(R_{\text {tip }}\right)^{0.5}$; This is obtained by approximating $F$ with $2 \beta V_{\mathrm{ge}} / D$, where $\beta$ is the field enhancement factor at the emitter apex proportional to $\sim 1 /\left(R_{\text {tip }}\right)^{0.5}$ for our pyramidal emitters. ${ }^{15}$

In Figure 2, the $I-V$ of FEA 2 denoted as $a$ was observed after conditioning at the base pressure in the preparation chamber. After subsequent conditioning in $4 \times 10^{-5}$ mbarAr gas for $5 \mathrm{~h}$ in total ( $b$ in Fig. 2(b)), $A_{\mathrm{FN}}$ became $13.3 \pm 3.1$ times higher and $B_{\mathrm{FN}}$ increased by $17 \% \pm 2 \%$. These values suggested an increase of $N R_{\text {tip }}{ }^{2}$ and an increase of $R_{\text {tip. }}$. It is difficult to account for an order of magnitude increase of $A_{\mathrm{FN}}$ and fractional increase of $B_{\mathrm{FN}}$ by sole variation of $W . I_{\mathrm{g}}$ ( $b$ ' in Fig. 2(b)) was below $9 \%$ in the $V_{\mathrm{ge}}$ range,

TABLE I. Summary of the Fowler-Nordheim parameters of $I-V \mathrm{~s}$ of FEA2 in Fig. 2(b).

\begin{tabular}{lcc}
\hline \hline Index & \multicolumn{1}{c}{$A_{\mathrm{FN}}(\mathrm{A})$} & $B_{\mathrm{FN}}(\mathrm{V})$ \\
\hline$A$ & $(0.779 \pm 0.091) \times 10^{4}$ & $647.27 \pm 5.01$ \\
$B$ & $(10.358 \pm 2.104) \times 10^{4}$ & $756.98 \pm 9.35$ \\
$C$ & $(8.741 \pm 1.868) \times 10^{4}$ & $677.83 \pm 8.35$ \\
$D$ & $(8.754 \pm 1.500) \times 10^{4}$ & $662.99 \pm 6.78$ \\
\hline \hline
\end{tabular}


therefore, it does not affect the interpretation. We then transferred the chip from the preparation chamber to the diode gun through ambient air. After reaching $1 \times 10^{-8}$ mbar, DC $V_{\text {ge }}$ conditioning was conducted for a day ( $c$ in Fig. 2(b)) and subsequently in $10^{-5}$ mbar-Ar gas for $30 \mathrm{~min}(d$ in Fig. 2(b)) before the imaging experiment. The comparison of $b$ and $d$ shows that, as a result of the transfer of the chip through air, $A_{\mathrm{FN}}$ changed by a factor of $0.84 \pm 0.29$ and $B_{\mathrm{FN}}$ decreased by $12 \% \pm 2 \%$ (we approximated $I_{\mathrm{a}}$ with $I_{e m}$ because of the small difference between the pulse current and $I_{\mathrm{em}}$ ). This suggested a reduction of $R_{\text {tip }}$ and a degradation of the beam homogeneity due to, for example, a partial oxidation of emitter tips in air.

It is an interesting question whether other gas has the same effect as argon. We, therefore, tested the effect of $\mathrm{Ne}$ gas. Using FEA 3 and examining evolution of $I-V \mathrm{~s}$ in the preparation chamber, we again observed increased FN parameters; Fig. 3(a) shows the variation of pressure, the maximum $I_{\mathrm{a}}$, the maximum $V_{\mathrm{ge}}$, and $A_{\mathrm{FN}}$ and $B_{\mathrm{FN}}$ in time under AC $50 \mathrm{~Hz} V_{\mathrm{ge}}$. FN parameters obtained from the fitting of $I$ $V \mathrm{~s}, a-f$ in Fig. 3(b), were summarized in Table II. After initial conditioning ( $a$ in Fig. 3), we repeatedly operated the chip under $10^{-4}$ mbar-Ne gas environment and at the base pressure. This resulted in the increase of $A_{\mathrm{FN}}$ and $B_{\mathrm{FN}}$ (the variation from $a$ to $b$ ). The periodical increase of $I_{\mathrm{a}}$ during this time resulted from a combination of decreasing $I_{\mathrm{a}}$ and subsequent manual increase of the maximum $V_{\text {ge }}$. A gradual decrease of $A_{\mathrm{FN}}$ and $B_{\mathrm{FN}}$ followed after returning to the base pressure (the variation from $b$ to $c$ ). Still, $A_{\mathrm{FN}}$ and $B_{\mathrm{FN}}$ of $c$ remained higher than $a$. After three additional cycles, the resultant $I-V\left(d\right.$ in Fig. 3) exhibited $A_{\mathrm{FN}}$ and $B_{\mathrm{FN}}$, respectively, $16.2 \pm 1.0$ times and $1.95 \pm 0.01$ times larger than those of $a$. We then tested high current operation of FEA3 in this state in the preparation chamber with 92-ns-long pulsed $V_{\text {ge }}$. The data, $e$ and $f$, in Fig. 3(b) show two scan results with different maximum $V_{\text {ge }}$. The $I_{\mathrm{a}}-V_{\text {ge }}$ of $e$ were approximately the same as $d$ but $f$ with maximum $V_{\text {ge }}$ of $160 \mathrm{~V}$ shifted to higher $V_{\text {ge }}$ and $A_{\mathrm{FN}}$ and $B_{\mathrm{FN}}$ increased as well. This $e$-to- $f$ variation of $I-V$ appeared to be due to current-induced tip-blunting, ${ }^{8,12}$

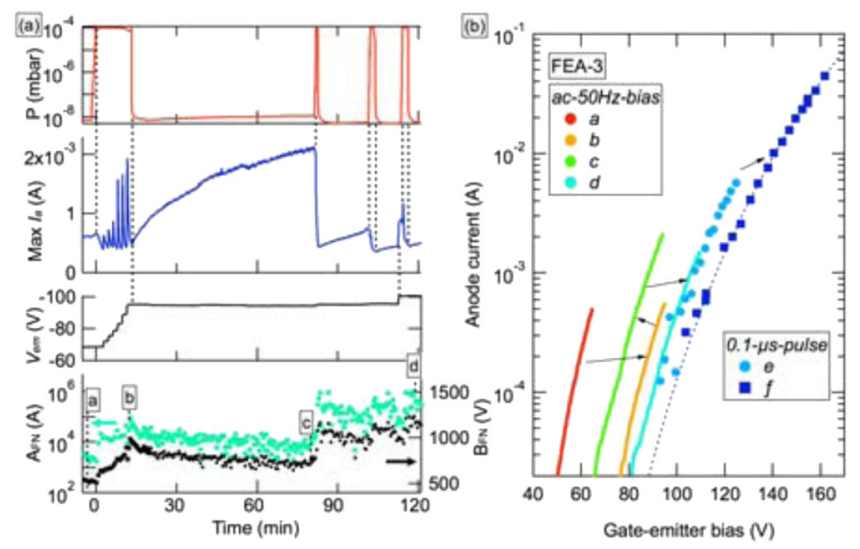

FIG. 3. (Color online) Variation of the maximum anode current $I_{\mathrm{a}}$, the maximum $V_{\mathrm{ge}}$ amplitude, pressure with Ne-gas, and FN parameters $A_{\mathrm{FN}}$ and $B_{\mathrm{FN}}$ under $50 \mathrm{~Hz}-\mathrm{AC}-V_{\mathrm{ge}}$ for FEA3. (b) Evolution of $I_{\mathrm{a}}-V_{\mathrm{ge}}$ characteristics. $a$ to $d$ were measured with $50 \mathrm{~Hz}-\mathrm{AC}-\mathrm{Vge}$. $e$ and $f$ were obtained from a pulsed measurement with 92 ns-FWHM $V_{\text {ge }}$ pulse.
TABLE II. Summary of the Fowler-Nordheim parameters of $I$-Vs of FEA3 in Fig. 3(b).

\begin{tabular}{lcc}
\hline \hline Index & $A_{\mathrm{FN}}(\mathrm{A})$ & $B_{\mathrm{FN}}(\mathrm{V})$ \\
\hline$A$ & $(0.303 \pm 0.012) \times 10^{4}$ & $485.6 \pm 1.59$ \\
$B$ & $(7.413 \pm 0.427) \times 10^{4}$ & $911.77 \pm 3.52$ \\
$C$ & $(1.026 \pm 0.024) \times 10^{4}$ & $696.29 \pm 1.34$ \\
$D$ & $(4.903 \pm 0.238) \times 10^{4}$ & $946.96 \pm 3.42$ \\
$E$ & $(2.109 \pm 0.917) \times 10^{4}$ & $890.38 \pm 35.7$ \\
$F$ & $(5.215 \pm 0.918) \times 10^{4}$ & $1048.5 \pm 16.4$ \\
\hline
\end{tabular}

but no arc was observed until $I_{\mathrm{a}}$ reached the maximum value of $45 \mathrm{~mA}$ at $160 \mathrm{~V}$. This current corresponded to $4.5 \mu \mathrm{A}$ per tip and was higher than $2.5 \mu \mathrm{A}$ per tip from a 1600-tip molded single-gate FEA reported previously. ${ }^{3,16}$

As one underlying mechanism of the emission homogeneity improvement, we consider that bombardment of emitters by noble-gas ions, that were generated by electron impact ionization near current-emitting tips, caused sputtering ${ }^{6}$ or ion-implantation. ${ }^{17}$ From the expected $R_{\text {tip }}$-dependences for $A_{\mathrm{FN}}\left(\sim N R_{\mathrm{tip}}{ }^{2}\right)$ and $B_{\mathrm{FN}}\left(\sim\left(R_{\mathrm{tip}}\right)^{0.5}\right)$ with the FN exponent $n$ of 2 , the observed increase of $A_{\mathrm{FN}}$ and $B_{\mathrm{FN}}$ was compatible with the increase of $R_{\text {tip }}$ by sputtering-induced tip-blunting and removal of adsorbate and native oxide. Preferential sputtering of oxygen atoms from partially oxidized molybdenum emitter surface could also contribute to this. ${ }^{18}$

The authors acknowledge B. Haas, J. Leemann, and A. Luecke for their help for FEA fabrication. This work was partially supported by Swiss National Science Foundation.

${ }^{1}$ S. Tsujino, M. Paraliev, E. Kirk, T. Vogel, F. Le Pimpec, C. Gough, S. Ivkovic, and H.-H. Braun, J. Vac. Sci. Technol. 29, 02B117(2011).

${ }^{2}$ S. Tsujino, M. Paraliev, E. Kirk, C. Gough, S. Ivkovic, and H.-H. Braun, Phys. Plasmas 18, 064502 (2011).

${ }^{3}$ E. Kirk, S. Tsujino, T. Vogel, K. Jefimovs, J. Gobrecht, and A. Wrulich, J. Vac. Sci. Technol. B 27, 1813 (2009).

${ }^{4}$ A. Mustonen, P. Beaud, E. Kirk, T. Feurer, and S. Tsujino, in 8th International Vacuum Electron Source Conference and Nanocarbon, Nanjing, 13-16 October, 2010; submitted to Appl. Phys. Lett. (2011).

${ }^{5}$ M. Ding, G. Sha, and A. I. Akinwande, IEEE Trans. Electron Devices 49, 2333 (2002).

${ }^{6}$ D. Nicolaescu, M. Nagao, T. Sato, V. Filip, S. Kanemaru, and J. Itoh, J. Vac. Sci. Technol. B 23, 707 (2005).

${ }^{7}$ K. L. Jensen, P. G. O'Shea, D. W. Feldman, and J. L. Shaw, J. Appl. Phys. 107, 014903 (2010).

${ }^{8}$ J. D. Jarvis, H. L. Andrews, C. A. Brau, B. K. Choi, J. Davidson, W.-P. Kang, and Y.-M. Wong, J. Vac. Sci. Technol. B 27, 2264 (2009).

${ }^{9}$ W. P. Dyke and J. K. Trolan, Phys. Rev. 89, 799 (1953).

${ }^{10}$ I. Brodie and C. A. Spindt, "Vacuum Microelectronics" in Advances in Electronics and Electron Physics, 83rd ed., edited by P. W. Hawkes (Academic, Boston, 1992), p. 6.

${ }^{11}$ Application of negative $V_{\text {ge }}$ to the emitter substrate minimized collection of current by gate during the conditioning in the diode gun.

${ }^{12}$ P. R. Schwoebel, C. A. Spindt, and C. E. Holland, J. Vac. Sci. Technol. B 23, 691 (2005).

${ }^{13}$ C. A. Spindt, I. Brodie, L. Humphrey, and E. R. Westerberg, J. Appl. Phys. 47, 5248 (1976).

${ }^{14}$ R. G. Forbes and J. H. B. Deane, Proc. R. Soc. A 463, 2907 (2007).

${ }^{15}$ S. Tsujino, F. le Pimpec, J. Raabe, M. Buess, M. Dehler, E. Kirk, J. Gobrecht, and A. Wrulich, Appl. Phys. Lett. 94, 093508 (2009).

${ }^{16} \sim 20 \mu$ A-per-tip current from a 7-tip device was reported in Ref. 3 as well.

${ }^{17}$ R. H. Reuss and B. R. Chalamala, J. Vac. Sci. Technol. B 21, 1187 (2003).

${ }^{18}$ J. B. Malherbe, S. Hofmann, and J. M. Sanz, Appl. Surf. Sci. 27, 355 (1986). 